\title{
Stress and Strain Rate Effects on Incipient Spall in Tantalum
}

\author{
David R. Jones ${ }^{1 *}$, Saryu J. Fensin ${ }^{1}$, Carl P. Trujillo ${ }^{1}$, Daniel T. Martinez ${ }^{1}$, and George T. Gray III ${ }^{1}$ \\ ${ }^{1}$ MST-8 Division, Los Alamos National Laboratory, Los Alamos, NM 87545, USA
}

\begin{abstract}
Spall fracture is a high-rate tensile damage phenomenon associated with impulsive and shock- load events. Typically, the material undergoes a sequence of compression followed by release into high rate (on the order of $10^{4} \mathrm{~s}^{-1}$ and up) tension, causing voids to nucleate and grow, which can then coalesce into a crack and the material fails. We present a series of experiments on high purity, well characterized tantalum samples subjected to shock-loading via gas-gun plate impact. Through careful selection of the flyer-plate velocity and material we have independent control over the peak compressive stress and the tensile strain rate in the sample. At all times, the spall damage remains incipient, i.e. in the early stages of void formation and the material does not fully fracture. Velocimetry was used on the rear of the sample to record the wave-profiles and determine spall strength. Soft recovery and sectioning of the samples allowed the internal damage to be observed, quantifying the damage amount, distribution, and relationship to microstructural features with both optical and electron based microscopy.
\end{abstract}

\section{Introduction}

Spall fracture is a high strain-rate phenomena associated with shock- or impulse-based loading, such as that from impact or explosive blast. In brief, the process begins with a sample being loaded to some stress. This stress is then relieved, allowing release or rarefaction waves to propagate into the sample. As these rarefactions accelerate material in the opposite direction to their propagation, where the two intersect the sample is driven into tension at a high rate, typically on the order of $10^{4} \mathrm{~s}^{-1}$ upwards [1]. This tension generates damage in a sequence of void nucleation, followed by growth, and finally coalescence, where if significant quantities occur the sample can split and produce a spall scab.

Tantalum has been extensively studied under dynamic loading conditions, due to its density, ductility, high melting point, and toughness [2]. The spall strength, a measure of a material's resistance to spall fracture, is typically on the order of $5 \mathrm{GPa}$ to $8 \mathrm{GPa}$ for tantalum, for peak stresses attainable on single-stage light gas-guns, around $20 \mathrm{GPa}$ [3]. It has also been shown that the spall strength reduces as the tensile pulse duration is increased [4]. The spall strength is calculated from the velocity history of the rear free-surface of a target. This presents challenges, as one must infer internal behavior from an external measurement, exacerbated by the statistical nature of void nucleation and the typically small surface area probed. Moving to two-dimensional diagnostics such as line VISAR have revealed large differences in the calculated spall strength as a function of sampling position [5].

\footnotetext{
*e-mail: djones@lanl.gov
}

While the spall strength is a useful quantity when comparing similar experiments, for a fully-predictive modeling capability, data is needed on where damage nucleates and how it is distributed in the sample. In this study, we aim to limit spall to the early stages of incipient damage, such that the sample remains intact and can be sectioned for post-mortem analyses. It will be demonstrated with a series of flyer-plate impact experiments that changing the loading and release conditions (via flyer-plate material and impact velocity) can result in drastically different damage patterns while producing an almost constant value for spall strength.

\section{Experimental Method}

\subsection{Material}

The tantalum was supplied as a large disc, approximately $50 \mathrm{~cm}$ in diameter and $1 \mathrm{~cm}$ thick, by H.C. Starck GmbH. A series of processing steps were used to ensure all samples sectioned from the plate had the same nominal microstructure. To examine the initial condition of the tantalum plate samples were prepared for electron microscopy. Electron backscatter diffraction (EBSD) techniques were used to determine grain size and texture. It was found that the microstructure was approximately equiaxed, with an average grain size of $30 \mu \mathrm{m}$, and a weak $\langle 100\rangle$ texture ( 5 times random), along the plate through-thickness direction. These results are shown in Figure 1. Samples for the experiments in section 2.2 were taken such that the loading direction was along the through-thickness direction of the plate. Both tantalum and single crystal z-cut quartz were used as flyer-plate materials, with material parameters given in Table 1. 
Table 1. Equation of state parameters for the materials used.

\begin{tabular}{lll}
\hline Parameter & Tantalum [6] & Z-cut Quartz [7] \\
\hline Density, $\rho_{0}$ & $16580 \mathrm{~kg} \mathrm{~m}^{-3}$ & $2650 \mathrm{~kg} \mathrm{~m}^{-3}$ \\
Shock Velocity Intercept, $C_{0}$ & $3430 \mathrm{~m} \mathrm{~s}^{-1}$ & $6360 \mathrm{~m} \mathrm{~s}^{-1}$ \\
$U_{s}-u_{p}$ Gradient, $s$ & 1.19 & 1.36 \\
Longitudinal Sound Speed, $C_{L}$ & $4130 \mathrm{~m} \mathrm{~s}^{-1}$ & - \\
Bulk Sound Speed, $C_{B}$ & $3392 \mathrm{~m} \mathrm{~s}^{-1}$ & - \\
\hline
\end{tabular}

\subsection{Plate-Impact Experiments}

All experiments were performed on an $80 \mathrm{~mm}$ bore singlestage light gas-gun. Disc shaped samples were machined from the large plate described in section $2.1,19.1 \mathrm{~mm}$ diameter by $4 \mathrm{~mm}$ thick. These were then pressed into a momentum ring assembly designed to mitigate the effects of edge release waves entering the sample and perturbing the one-dimensional loading state. This arrangement has been discussed in detail by Gray [8, ch. 6] and Bourne [9]. A stripper-plate and catch-can filled with soft-recovery media was used to separate the samples from the momentum rings and arrest the projectile, allowing the sample to be gently captured for post-mortem analysis.

To have control over the rate of release into tension, two different flyer-plate materials were used. As this rate of release is determined by the release isentrope of the material, and therefore the width of the rarefaction fan, z-cut quartz was chosen for the high strain-rate condition. At the stresses in this study the quartz flyer-plate remains elastic [10], and hence has a very narrow rarefaction fan and a subsequently high release rate. For the lower strainrate case, the same tantalum as the targets was used, for a
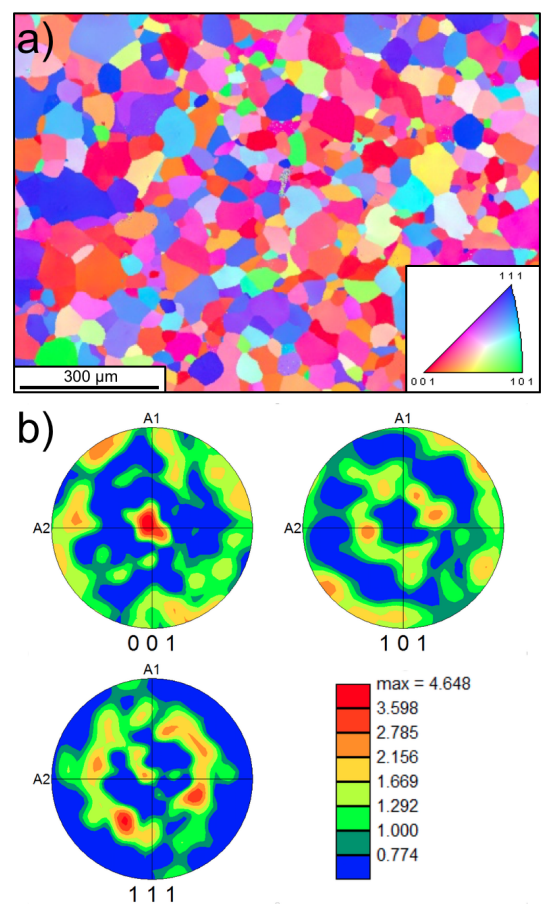

Fig. 1. a) Inverse pole-figure map of the tantalum plate used for the samples, viewing along the through-thickness direction. b) Pole figures of the above map showing a mild $\langle 100\rangle$ texture. symmetric impact condition. This has a much wider rarefaction fan, corresponding to a more gentle rate of release into tension. To ensure the shock duration and location of the spall plane center was the same for the two flyer-plate scenarios, sound wave speeds were used to calculate the flyer-plate thicknesses as $1.05 \mathrm{~mm}$ for the tantalum case and $2 \mathrm{~mm}$ for the quartz. These values produced a shock duration of approximately $0.7 \mu \mathrm{s}$.

The peak stress in the sample was controlled by adjusting the impact velocity of the flyer-plate onto the sample. The parameters in Table 1 were used along with the Rankine-Hugoniot jump conditions to calculate the peak stress:

$$
\begin{aligned}
\sigma & =\rho_{0} U_{s} u_{p}, \\
U_{s} & =C_{0}+s u_{p},
\end{aligned}
$$

where $\sigma$ is stress, $\rho_{0}$ is initial density, $U_{s}$ is shock velocity, $u_{p}$ is particle velocity, and $C_{0}$ and $s$ are the fit-values for the linear shock equation of state. The tensile strainrate was calculated with the approximation:

$$
\dot{\varepsilon}=\frac{1}{2} \frac{\dot{u_{1}}}{C_{B}}
$$

where $u_{1}$ is the slope of the free-surface velocity pullback section, and $C_{B}$ is the material's bulk sound speed. The factor of $1 / 2$ results from the approximation that the particle velocity is half the free-surface velocity. It is important to note that this is a simple, although widely used, approximation; it is useful for comparison between samples, assuming that any errors are systematic and similar for all experiments [11]. From the columns for peak stress and strain-rate in Table 2 it is clear that the four experiments can be grouped into two low stress and two high stress shots, with a low strain-rate and a high strain-rate example in each.

Diagnostics consisted of a multiple-channel frequency-shifted photon Doppler velocimetry (PDV) system [12]. Each experiment used three probes: one to measure projectile velocity, one at the center of the rear free-surface of the target, and one $3 \mathrm{~mm}$ off-center. Velocity histories were reduced from the PDV data using the conventional short-time Fourier transform techniques [13].

\subsection{Damage Quantification}

The recovered samples were diametrically sectioned and cast in epoxy, such that the internal face normal to the shock direction could be prepared for microscopy. A series of grinding and polishing steps were completed, followed by an acid etch to highlight microstructural features. 
Table 2. Details of the plate-impact experiments.

\begin{tabular}{cccccc}
\hline \multirow{2}{*}{ Experiment } & Conditions & Flyer-Plate & $\begin{array}{c}\text { Flyer Velocity } \\
\left(\mathrm{m} \mathrm{s}^{-1}\right)\end{array}$ & $\begin{array}{c}\text { Peak Stress } \\
(\mathrm{GPa})\end{array}$ & $\begin{array}{c}\text { Tensile Strain-Rate } \\
\left(\mathrm{s}^{-1}\right)\end{array}$ \\
\hline$\# 6$ & Low Stress, Low Rate & Tantalum, 1.05 mm & $196 \pm 2$ & 5.8 & $6.0 \times 10^{4}$ \\
$\# 2$ & Low Stress, High Rate & Quartz, 2 mm & $417 \pm 4$ & 5.8 & $8.1 \times 10^{4}$ \\
$\# 4$ & High Stress, Low Rate & Tantalum, 1.05 mm & $214 \pm 2$ & 6.3 & $6.0 \times 10^{4}$ \\
$\# 5$ & High Stress, High Rate & Quartz, 2 mm & $451 \pm 4$ & 6.3 & $8.7 \times 10^{4}$ \\
\hline
\end{tabular}

Optical micrographs were imported to the software package ImageJ, where a thresholding function was applied to create a binary image. Here, voids or damage correspond to a pixel value of 1 and the surrounding tantalum is 0 . Knowing the scale of the micrographs $(10 \mathrm{~mm}$ wide by $3 \mathrm{~mm}$ high), combined with the analyze particles routine in ImageJ, allows certain damage metrics to be extracted. Specifically, the number, size, and circularity of each void is measured. As the spall fracture process is statistical in nature, each sample was prepared and imaged four times at subsequent depths approximately $150 \mu \mathrm{m}$ apart. Errors in the damage measurements are based on the variation between these slices.

\section{Results}

\subsection{Free-Surface Velocity Data}

The free-surface velocity histories for the center-probe for each sample are shown in figure 2. The two low-stress $(5.8 \mathrm{GPa})$ experiments are marked with a solid line, and the two high-stress $(6.3 \mathrm{GPa})$ experiments with a dotted line. Black traces correspond to the tantalum flyer-plate, low strain-rate samples, with the red traces being the quartz flyer-plate, high strain-rate samples. The traces have been shifted in time such that shock-breakout is at the same arbitrary time for each trace.

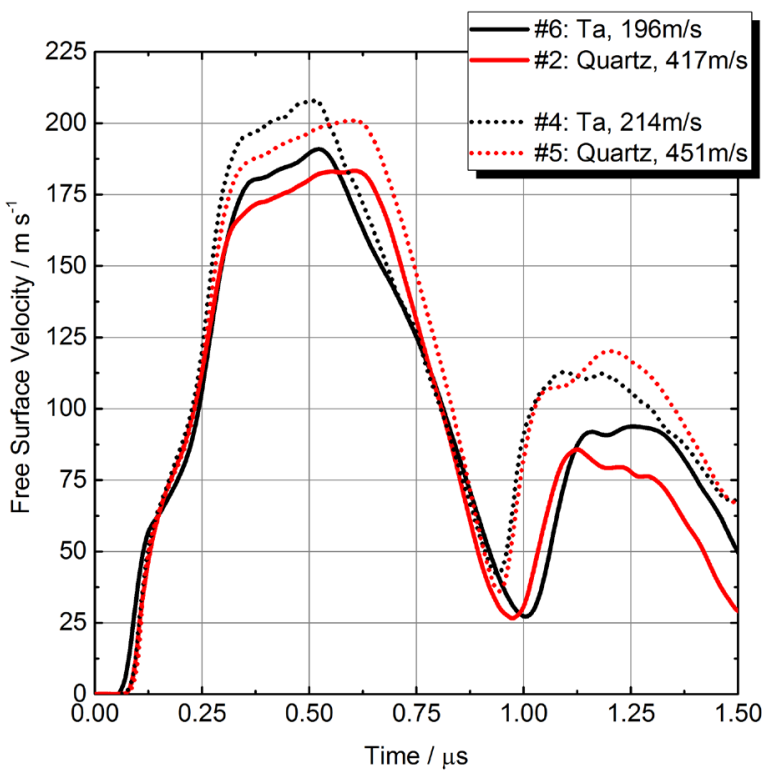

Fig. 2. Free surface velocity profiles for the plate-impact experiments. Legend describes the flyer-plate and impact velocity.
All four experiments show the classic flyer-plate impact and spall response. First, there is an elastic precursor, on the order of $60 \mathrm{~m} \mathrm{~s}^{-1}$, corresponding to a Hugoniot Elastic Limit (HEL) of 2.1 GPa. Due to the relatively low impact velocities and stresses in this study, in all cases the elastic wave is a significant fraction of the plastic wave magnitude. As a result of this, as the elastic wave reaches the rear free surface first and reflects back towards the later plastic wave, it attenuates the typical 'flat-topped' shock wave profile, causing the sloped appearance of the peak free surface velocities. This is more evident in the hydrocode simulations discussed in section 4.1.

After the peak-state, there is a deceleration phase as the sample enters into tension. This reaches some minimum, the difference between this and the peak-state and this relates to the level of tensile stress the sample can endure before damage nucleates. This internal damage grows and presents at the rear free-surface as an acceleration. The gradients and magnitude of this pullback were used to calculate the spall strength for each sample with the following equations:

$$
\begin{gathered}
\sigma_{\mathrm{sp}}=\frac{1}{2} \rho_{0} c_{B}\left(\Delta u_{\mathrm{fs}}+\delta\right), \\
\delta=\left(\frac{h_{\mathrm{sp}}}{c_{B}}-\frac{h_{\mathrm{sp}}}{c_{L}}\right) \cdot \frac{\left|\dot{u_{1}}\right| \cdot\left|\dot{u}_{2}\right|}{\left|\dot{u}_{1}\right|+\left|\dot{u}_{2}\right|},
\end{gathered}
$$

where $\Delta u_{\mathrm{fs}}$ is pullback in the free surface velocity after the peak state, $h_{\mathrm{sp}}$ is the distance from the spall plane to the rear free surface and $\dot{u}_{1}$ and $\dot{u}_{2}$ are the gradients of the deceleration and acceleration phase of the spall pullback signal. Equation 5 is used to correct for the issues caused by using a free surface measurement to quantify an internal process [14]. The values for the pullback features and spall strengths for each experiment are given in Table 3.

In general, the spall strength increased with peak stress. It has been widely observed that as stresses and strain-rates increase in experiments, the spall strength of tantalum (and many other matierals) increases [15], as the reduction in timescales inhibits traditional methods of plasticity and strengths tend towards theoretical maxima [16]. However, there does not appear to be a strong trend in the spall strength when comparing different strainrates at the same peak stress (i.e. comparing the tantalum and quartz flyer-plates, \#6 against \#2, or \#4 against \#5). As previously mentioned, it is expected that spall strength will increase with strain-rate. While this is the case in experiments \#4 and \#5, the trend reverses in the other pair (\#6 vs. \#2). At these low impact velocities and stresses, the uncertainties in the PDV data can be non-negligible when compared to the peak velocities, and as such any trends will be difficult to discern. 
Table 3. Spall strength, damage quantities and distributions.

\begin{tabular}{ccccccc}
\hline Experiment & Conditions & $\begin{array}{c}\text { Spall Strength } \\
(\mathrm{GPa})\end{array}$ & $\begin{array}{c}\text { Number } \\
\text { of Voids }\end{array}$ & $\begin{array}{c}\text { Damaged } \\
\text { Area }(\%)\end{array}$ & $\begin{array}{c}\text { Mean Void } \\
\text { Diameter }(\mu \mathrm{m})\end{array}$ & $\begin{array}{c}\text { Spall Region Width } \\
(\mu \mathrm{m})\end{array}$ \\
\hline$\# 6$ & Low Stress, Low Rate & $5.1 \pm 0.2$ & $406 \pm 23$ & $1.4 \pm 0.1$ & $35 \pm 1$ & $197 \pm 11$ \\
$\# 2$ & Low Stress, High Rate & $4.9 \pm 0.2$ & $510 \pm 24$ & $0.8 \pm 0.1$ & $24 \pm 1$ & $397 \pm 30$ \\
$\# 4$ & High Stress, Low Rate & $5.2 \pm 0.2$ & $541 \pm 36$ & $3.9 \pm 0.3$ & $52 \pm 2$ & $222 \pm 6$ \\
$\# 5$ & High Stress, High Rate & $5.3 \pm 0.2$ & $635 \pm 45$ & $0.7 \pm 0.1$ & $20 \pm 1$ & $441 \pm 22$ \\
\hline
\end{tabular}

\subsection{Damage}

Optical images of the damaged region for each sample are shown in Figure 4. There is a clear difference between the two tantalum flyer-plate low strain-rate experiments (top row, \#6 and \#4) and the quartz flyer-plate high strain-rate experiments. In the former, the amount of damage is much greater than the latter, and is more localized into a thin band. As the peak stress is increased this band becomes more defined as voids have progressed from the nucleation and growth phase into coalescence with neighboring voids. In contrast, the quartz-driven experiments have far less overall damage, albeit spread over a wider region, with voids remaining isolated and circular.

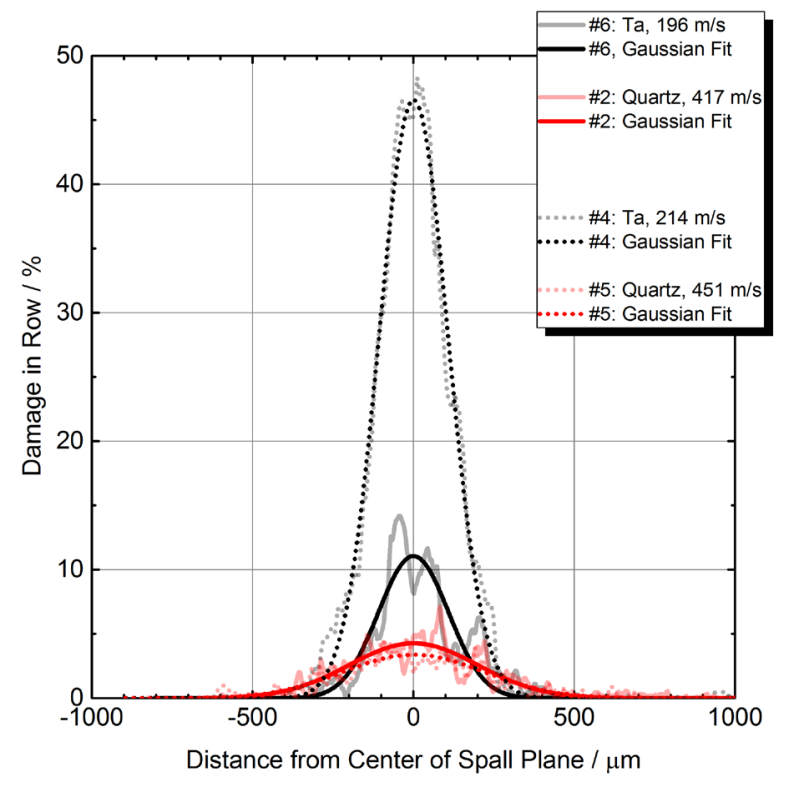

Fig. 3. Damage distribution around the spall plane, with the Gaussian fits overlaid. All plots have been shifted to be centered at zero distance to facilitate comparison between experiments.

The values in Table 3 represent the average of the multiple analyses performed on each sample. These values confirm the finding that there is a greater number of voids in the quartz flyer cases, which have remained small and isolated when compared with the tantalum flyer-plate experiments. The average circularity of the voids for both quartz cases was 0.96 , dropping to 0.93 and 0.91 for the tantalum cases (in order of increasing peak stress) as the amount of coalescence increases. The final column of Table 3 , spall region width, is a measure of how widely distributed the damage is in each sample. To calculate this, the total amount of damage in each row of the binary images was summed. The total for each row was then plotted against position in the sample, and a Gaussian curve was fitted to the distribution of damage vs. position. The fullwidth-at-half-maximum for the fitted Gaussian was taken as the spall region width. A plot of this damage distribution and fitted curves for a single slice of each sample is shown in Figure 3, where the plots have been shifted such that the center of the distributions are at zero distance.

\section{Discussion}

The most striking difference between the experiments is the greater amount of damage, and how it is much more concentrated, in the two tantalum flyer-plate experiments. At first glance this is somewhat counter-intuitive; it would make sense that the quartz flyer-plate, with a far narrower rarefaction fan, would result in a more localized damage distribution. Instead, the opposite was observed. Furthermore, within uncertainties, the calculated spall strengths were the same for all drive conditions. This highlights the need for incipient damage experiments and post-mortem characterization to compliment the free-surface velocity data, as it is clear that the samples from experiments \#4 and \#5 would have a very different response if subjected to a second load, or expected to perform under tension.

Our hypothesis for the different damage distributions is related to how the spread of the rarefaction fans originating in the flyer-plate (and the sample, but this is constant between these experiments) affects both the volume of sample pulled into tension, and the time spent under tension. As the two colliding fans intersect, the stress in the sample will be reduced from compression, through zero, and into tension. In the case where the fan has a large spread, as with the tantalum flyer-plate, this process takes longer to distribute throughout the entire sample, instead remaining in a smaller region. Hence the tensile stress is more concentrated, there is less material volume to accommodate the plasticity resulting from the stress, and a narrow band of extreme damage will form. In the quartz flyerplate experiments, while the applied tensile stress will be very similar to that in the tantalum cases, the stress is more quickly distributed across a larger volume of material, and the damage remains in the void nucleation phase. To demonstrate this graphically, hydrocode simulations were performed.

\subsection{Hydrocode Simulations}

Lagrangian two-dimensional axi-symmetric simulations of experiments \#4 and \#5 were performed in the hydrocode 


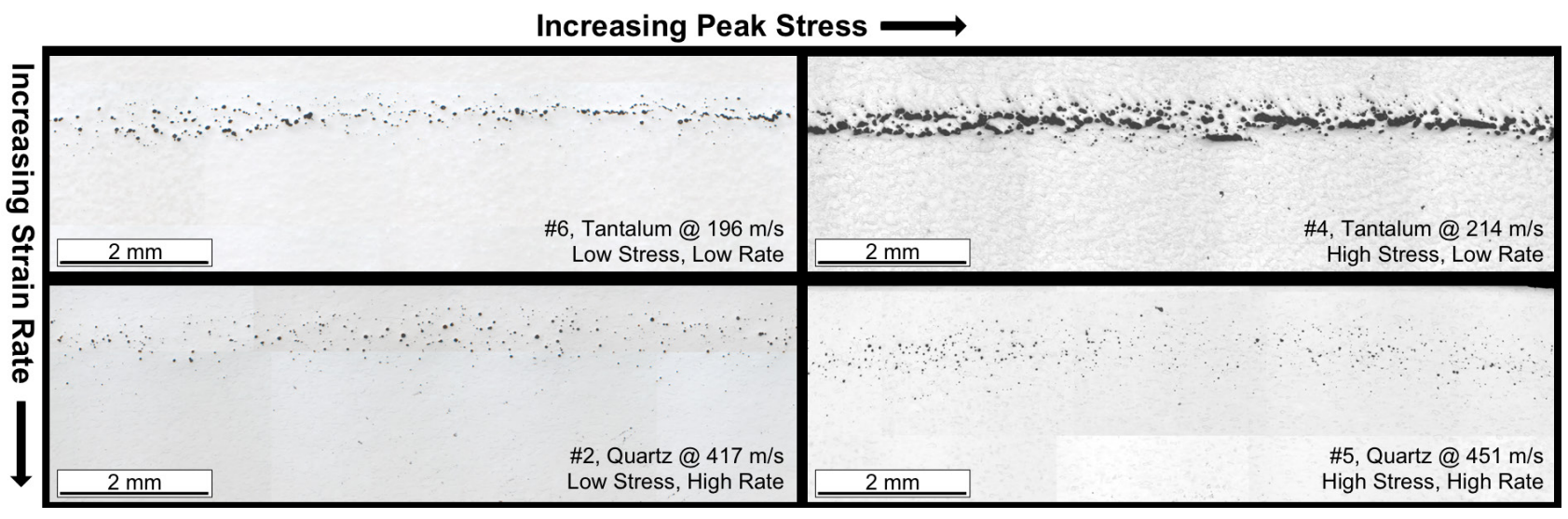

Fig. 4. Optical micrographs, $5 \mathrm{x}$ magnification, of the four recovered samples. Shock direction for all images is bottom-to-top.

CTH [17]. The outputs of these are shown in Figure 5, in the form of an $x-t$ (position-time) diagram. In these, the position is given along the $x$ axis, where the flyerplate is travelling from left to right. Time increases up the $y$ axis, and the color or $z$ axis corresponds to the longitudinal stress in the system, along the $x$ axis. No damage or fracture model was used.

It is clear from the plots in Figure 5 that the narrow release fan in the quartz flyer-plate (plot a) results in a narrower fan in the tantalum sample. This results in a wider region of the sample being pulled into tension more quickly. In contrast, in plot b, showing the tantalum flyerplate case, it is shown that the wide fans from both surface releases lead to a more gradual pull into tension concentrated into a narrow region. This is exacerbated by the fact that the tantalum flyer-plate remains in contact with the sample, where the quartz flyer-plate rebounds and separates. The extra thickness of the attached tantalum flyerplate increases the time it takes for the waves to reverberate in the system. The end result is the tantalum-driven sample has the tensile stress concentrated in a smaller region, for a longer dwell time, leading to the narrower region of increased damage when compared to the quartz case.

\section{Conclusions}

Incipient spall tests have been conducted on well characterized, high-purity tantalum samples. Flyer-plates of tantalum and z-cut single crystal quartz were used to control the rate of release into tension in the sample, via the spread of their respective rarefaction fans. The peak stresses in the samples were controlled through adjustment of the impact velocity. A combination of surface velocimetry and post-mortem microscopy were used to calculate the spall strength for each experiment and characterize the damage distribution in the sample. Tensile strain rates were varied from $6 \times 10^{4} \mathrm{~s}^{-1}$ to $9 \times 10^{4} \mathrm{~s}^{-1}$ and peak stresses from $5.8 \mathrm{GPa}$ to $6.3 \mathrm{GPa}$. Over these ranges the spall strengths of all samples were very similar, at approximately $5 \mathrm{GPa}$.

However, after sectioning, both the damage quantity and distribution were markedly different between the quartz and tantalum driven samples. In the former, the amount of damage was low (both under 1\%). There was a larger number of voids, but they remained small and isolated, spread over a region twice as wide as the tantalum case. The average void diameter for the quartz driven samples was $22 \mu \mathrm{m}$, compared with the tantalum driven cases at $44 \mu \mathrm{m}$. The damage in the tantalum driven samples was primarily located in a thin band, and had gone through the void nucleation and growth phases into significant coalescence.

The reasoning behind this is believed to be the width of the rarefaction fans responsible for releasing the sample

a) $2 \mathrm{~mm}$ Quartz Flyer, $450 \mathrm{~m} / \mathrm{s}$

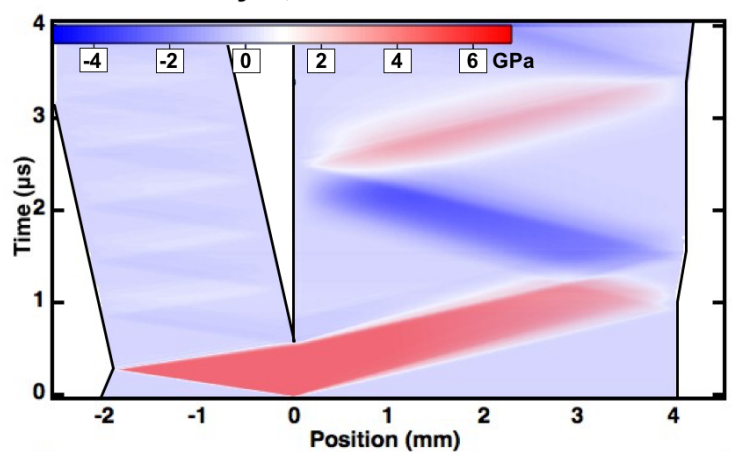

b) $1.05 \mathrm{~mm}$ Tantalum Flyer, $214 \mathrm{~m} / \mathrm{s}$

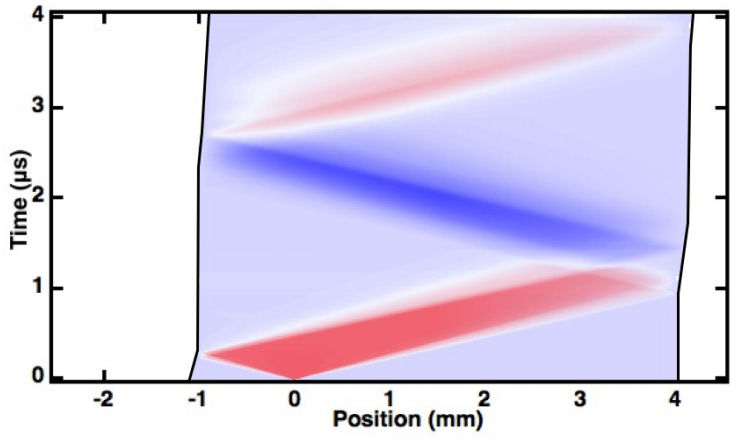

Fig. 5. $x-t$ (position-time) diagram produced from the CTH simulations for experiments \#5 (a) and \#4 (b) respectively. The color scale corresponds to the stress along the impact ( $+\mathrm{x}$ axis) direction. No fracture model was implemented. 
from the peak stress state. In the quartz flyer-plate, the fan is extremely narrow. This has the effect of pulling a large volume of the sample into a high level of tension quickly. As such, the plasticity is accrued in a large amount of material. Furthermore, as strain-rates increase, so to does the strength of the tantalum. This results in a relatively low amount of damage, restricted to void nucleation and minimal growth, dispersed over a large volume. In the tantalum flyer-plate experiments the opposite is found, where the wide rarefaction fans produce a narrow volume in tension for a longer duration. This causes the nucleated voids to be concentrated in a smaller volume, and allows the damage to progress to the coalescence phase.

This work highlights the issues with using simple spall strength based failure models, as even though the spall strength and free-surface velocity histories for each experiment were very similar, the internal damage was completely different. For a full predictive capability the entire loading history of the sample needs to be accurately captured. Future efforts will concentrate on flyer-plates with different rarefaction fan widths while keeping the peak stresses constant, to further investigate how the damage quantity and distribution is affected by interaction volume and dwell times.

Rob Hixson is thanked for many useful discussions and assistance with the simulations. Los Alamos National Laboratory is operated by Los Alamos National Security, LLC, for the National Nuclear Security Administration of the U.S. Department of Energy under contract DE-AC5206NA25396. Funding for this work was provided by the U.S. Department of Energy under the Laboratory Directed Research and Development (LDRD) program, Science Campaign 2, and the U.S. DoE / DoD Joint Munitions Program. Unlimited Release: LA-UR-18-21418.

\section{References}

1. G.I. Kanel, Int. J. Fract. 163, 173 (2010)

2. G.T. Gray III, A.D. Rollett, The High-Strain Rate and Spallation Response of Tantalum, Ta-10W and T-111, LA-UR-91-3160 (Los Alamos National Laboratory, Los Alamos, NM, 1991)
3. A.K. Zurek, W.R. Thissell, J.N. Johnson, D.L. Tonks, R. Hixson, Journal of Materials Processing Technology 60, 261 (1996)

4. J.C.F. Millett, G. Whiteman, N.T. Park, S. Case, N.K. Bourne, J. Appl. Phys. 113, 233502 (2013)

5. M.D. Furnish, L.C. Chhabildas, W.D. REinhart, W.M. Trott, T.J. Vogler, International Journal of Plasticity 25, 587 (2009)

6. S.P. Marsh, LASL Shock Hugoniot Data, Los Alamos Series on Dynamic Material Properties (University of Califonia Press, Berkeley, CA, 1980)

7. J. Wackerle, J. Appl. Phys. 33, 922 (1962)

8. J.R. Asay, M. Shahinpoor, eds., High Pressure Shock Compression of Solids (Springer-Verlag, New York, 1993)

9. N.K. Bourne, G.T. Gray III, Proceedings of the Royal Society A: Mathematical, Physical and Engineering Sciences 461, 3297 (2005)

10. R. Fowles, J. Geophys. Res. 72, 5729 (1967)

11. W.D. Turley, S.J. Fensin, R.S. Hixson, D.R. Jones, B.M. La Lone, G.D. Stevens, S.A. Thomas, L.R. Veeser, J. Appl. Phys. 123, 055102 (2018)

12. O.T. Strand, D.R. Goosman, C. Martinez, T.L. Whitworth, W.W. Kuhlow, Rev. Sci. Inst. 77, 083108 (2006)

13. T. Ao, D.H. Dolan, SIRHEN: a data reduction program for photonic Doppler velocimetry measurements, SAND2010-3628 (Sandia National Laboratories, Albuquerque, NM, 2010)

14. S.A. Novikov, Journal of Applied Mechanics and Technical Physics 22, 385 (1981)

15. E.N. Hahn, T.C. Germann, R. Ravelo, J.E. Hammerberg, M.A. Meyers, Acta Mat. 126, 313 (2017)

16. S.I. Ashitkov, P.S. Komarov, E.V. Struleva, M.B. Agranat, G.I. Kanel, K.V. Khishchenko, J. Phys.: Conf. Ser. 653, 012001 (2015)

17. J.M. McGlaun, S.L. Thompson, Int. J. Imp. Eng. 10, 351 (1990) 